\title{
Cellular Energy Efficiency Evaluation Framework
}

\author{
(Invited Paper) \\ Gunther Auer*, Vito Giannini ${ }^{\dagger}$, István Gódor ${ }^{\ddagger}$, Per Skillermark ${ }^{\S}$, Magnus Olsson ${ }^{\S}$, \\ Muhammad Ali Imran $₫$, Dario Sabella", Manuel J. Gonzalez ${ }^{* *}$, Claude Desset ${ }^{\dagger}$, Oliver Blume ${ }^{\dagger \dagger}$ \\ *DOCOMO Euro-Labs, 80687 Munich, Germany, Email: auer@docomolab-euro.com \\ ${ }^{\dagger}$ IMEC, Leuven, Belgium \\ ${ }_{\ddagger}^{\ddagger}$ Ericsson Research, Budapest H-1117, Hungary \\ $\S$ Ericsson Research, SE-164 80 Stockholm, Sweden \\ " CCSR University of Surrey, Guildford GU2 7XH, UK \\ $\|_{\text {Telecom Italia, } 10148 \text { Turin, Italy }}$ \\ ** TTI (Technologies of Telecommunication and Information), 39011 Santander, Spain \\ ${ }^{\dagger \dagger}$ Alcatel-Lucent, Bell Labs Germany, 70435 Stuttgart, Germany
}

\begin{abstract}
In order to quantify the energy savings in wireless networks, the power consumption of the entire system needs to be captured and an appropriate energy efficiency evaluation framework must be defined. In this paper, the necessary enhancements over existing performance evaluation frameworks are discussed, such that the energy efficiency of the entire network comprising component, node and network level contributions can be quantified. The most important addendums over existing frameworks include a sophisticated power model for various base station (BS) types, which maps the RF output power radiated at the antenna elements to the total supply power of a BS site. We also consider an approach to quantify the energy efficiency of large geographical areas by using the existing small scale deployment models along with long term traffic models. Finally, the proposed evaluation framework is applied to quantify the energy efficiency of the downlink of a 3GPP LTE radio access network.

Index Terms - energy efficiency, green radio, power \& traffic model, system level energy efficiency simulations, energy aware radio and network technologies (EARTH)
\end{abstract}

\section{INTRODUCTION}

The global mobile communication industry is growing rapidly. Today there are already more than 4 billion mobile phone subscribers worldwide [1], more than half the entire population of the planet. Obviously, this growth is accompanied by an increased energy consumption of mobile networks. Global warming and heightened concerns for the environment of the planet require a special focus on the energy efficiency of these systems [2-4]. The EARTH ${ }^{1}$ project $[3,5]$ is a concerted effort to achieve this goal and as part of its objectives, a holistic framework is developed to evaluate and compare the energy efficiency of several design approaches of wireless cellular communication networks.

For the quantification of energy savings in wireless networks, the power consumption of the entire system needs to be captured and an appropriate energy efficiency evaluation framework $\left(\mathrm{E}^{3} \mathrm{~F}\right)$ is to be defined. The EARTH $\mathrm{E}^{3} \mathrm{~F}$ presented

${ }^{1}$ EU funded research project EARTH (Energy Aware Radio and neTwork tecHnologies), FP7-ICT-2009-4-247733-EARTH, Jan. 2010 to June 2012. https://www.ict-earth.eu in Section 2 provides the key levers to facilitate the assessment of the overall energy efficiency of cellular networks over a whole country. The $\mathrm{E}^{3} \mathrm{~F}$ primarily builds on well-established methodology for radio network performance evaluation developed in 3GPP; the most important addendums, introduced in Sections 3 and 4, are to add a sophisticated power model of the base stations (BSs) as well as a large-scale long-term traffic model extension to existing 3GPP traffic scenarios. Then, in Section 5 the $\mathrm{E}^{3} \mathrm{~F}$ is applied in order to provide an assessment of the BS energy efficiency of a 3GPP LTE network deployed within an average European country.

\section{ENERGy EFFICIENCY EVALUATION FrameWORK $\left(\mathrm{E}^{3} \mathrm{~F}\right)$}

The widely accepted state-of-the-art to evaluate the performance of a wireless network is to simulate the relevant aspects of the radio access network (RAN) at system level. The computed results are, e.g. the system throughput measured in bit/s, quality of service (QoS) metrics, and fairness in terms of cell-edge user throughput. In order to ensure that the results generated by different RAN system simulation tools are comparable, well defined reference systems and scenarios are specified. This is an outcome of extensive consensus work from standardization bodies, such as 3GPP [6], and international research projects, such as the EU project Wireless World Initiative New Radio (WINNER) [7], with partners from academia as well as from industry. The most recent example is the global effort in ITU to evaluate system proposals for compliance with IMT-Advanced requirements [8]. In that direction, the EARTH $E^{3} \mathrm{~F}$ builds on the 3GPP evaluation framework for LTE [6].

Fig. 1 shows the necessary enhancements over existing performance evaluation frameworks, such that the energy efficiency of the entire network, comprising component, node and network level, over an extended time frame can be quantified. The EARTH $\mathrm{E}^{3} \mathrm{~F}$ illustrated in Fig. 1, identifies the essential building blocks that are necessary for an accurate holistic assessment of energy efficiency enhancements. Although the specific realization of a system level simulation tool largely 


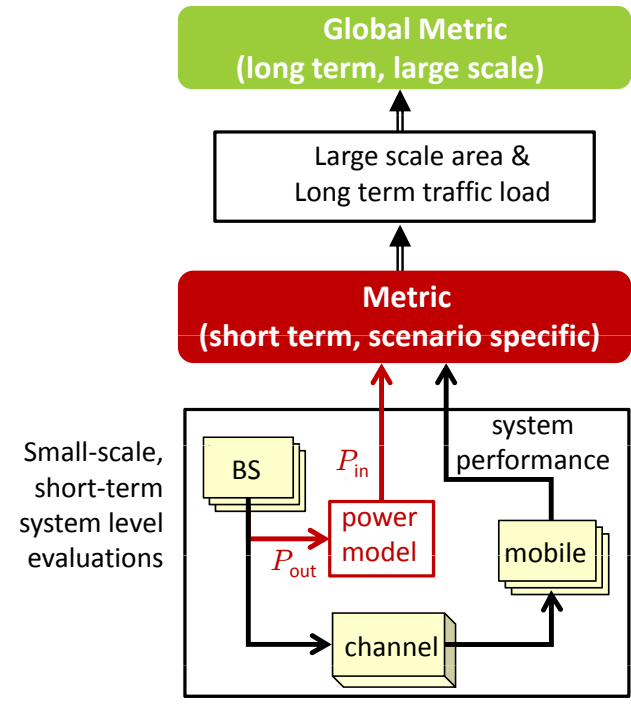

Fig. 1. EARTH Energy efficiency evaluation framework $\left(E^{3} F\right)$.

depends on the specific problem at hand, as well as the chosen software implementation, it is envisaged that for the assessment of combinations of energy efficiency enhancements integrated into one holistic system concept, the $\mathrm{E}^{3} \mathrm{~F}$ should capture the following aspects:

- A sophisticated power model (specified in Section 3), that maps the RF output power radiated at the antenna elements to the total supply power of a BS site. The power model maps the gains on the component level (e.g. an improvement of the energy efficiency of the power amplifiers) to energy savings on the entire network.

- Long-term traffic models (established in Section 4), that describe load fluctuations over a day and complement the statistical short-term traffic models.

- Large-scale deployment models (developed in Section 4) of large geographical areas are considered to extend the existing small-scale deployment scenarios.

\section{A. Small-Scale, Short-Term System Level Evaluations}

Statistical traffic models (e.g. FTP file download or VoIP calls), specific small-scale deployment scenarios (e.g. urban macro-cell consisting of 57 hexagonal cells with uniformly distributed users), and power models that quantify the power consumption of components within a node, constitute smallscale, short-term system level evaluations (bottom block in Fig. 1). The small-scale, short-term system level evaluations are carried out by a system level simulation platform, augmented by a model capturing the BS power consumption.

\section{B. Global $E^{3} F$}

In order to extend small-scale, short-term evaluations to a global scale, covering countrywide geographical areas and ranging over a full day or week, long-term traffic models and large-scale deployment maps are to be integrated into the $\mathrm{E}^{3} \mathrm{~F}$, as illustrated in Fig. 1. The global assessment of network energy efficiency comprises the following steps:
1) Small-scale, short-term evaluations are conducted for all scenarios (dense urban, urban, suburban and rural) and for a representative set of traffic loads, which captures the range between the minimum and the maximum load observed in a certain deployment.

2) The system level evaluations provide energy consumption and other performance metrics (e.g. throughput, QoS) for each small-scale deployment and a certain traffic load.

3) Given the daily/weekly traffic profile of each deployment, the power consumption over a day/week is generated by weighted summing of the short-term evaluations.

4) Finally, the mix of deployment scenarios that quantify the area covered by cities, suburbs, highways and villages, yield the global set of the large-scale system energy consumption.

\section{POWER MODEL}

This section provides a power model for various types of LTE Base Stations. The power model constitutes the interface between component and system level, which allows quantifying how energy savings on specific components enhance the energy efficiency at the node and network level.

\section{A. Base Station Power Consumption Breakdown}

A BS site consists of multiple transceivers (TRXs). A TRX comprises an Antenna Interface (AI), a Power Amplifier (PA), a Radio Frequency (RF) small-signal transceiver section, a baseband (BB) interface including a receiver (uplink) and transmitter (downlink) section, a DC-DC power supply, an active cooling system, and an AC-DC unit (mains supply) for connection to the electrical power grid. In the following the various TRX parts are analyzed.

Antenna Interface $(A I)$ : The influence of the antenna type on power efficiency is modeled by a certain amount of losses, including the feeder (where relevant), antenna bandpass filters, duplexers, and matching components.

Power Amplifier (PA): Typically, the most efficient PA operating point is close to the maximum output power (near saturation). Unfortunately, non-linear effects and OFDM modulation with non-constant envelope signals force the power amplifier to operate in a more linear region, i.e., 6 to $12 \mathrm{~dB}$ below saturation [9]. This prevents Adjacent Channel Interference (ACI) due to non-linear distortions, and therefore avoids performance degradation at the receiver. However, this high operating backoff gives rise to poor power efficiency, which translates to a high power consumption $P_{\mathrm{PA}}$. Digital techniques such as clipping and digital pre-distortion $[10,11]$ in combination with Doherty PAs [9] improve the power efficiency and linearizes the PA, while keeping ACI under control, but require an extra feedback for pre-distortion and significant additional signal processing [11]. While these techniques are necessary in macro and micro BSs, they are not used in smaller BSs, as the PA power consumption accounts for a smaller percentage of the power breakdown, allowing for a higher operating back-off. 
The Small-Signal RF Transceiver (RF-TRX) comprises a receiver and a transmitter for uplink (UL) and downlink (DL) communication. The linearity and blocking requirements of the RF-TRX may differ significantly depending on the BS type, and so its architecture. Typically, low-IF (IntermediateFrequency) or super-heterodyne architectures are the preferred choice for macro/micro BSs, whereas a simpler zero-IF architecture are sufficient for pico/femto BSs [12]. Parameters with highest impact on the RF-TRX energy consumption, $P_{\mathrm{RF}}$, are the required bandwidth, the allowable Signal-to-Noise And Distortion ratio (SiNAD), the resolution of the analogue-todigital conversion, and the number of antenna elements for transmission and/or reception.

Baseband (BB) Interface: The baseband engine (performing digital signal processing) carries out digital up/downconversion, including filtering, FFT/IFFT for OFDM, modulation/demodulation, digital-pre-distortion (only in DL and for large BSs), signal detection (synchronization, channel estimation, equalization, compensation of RF non-idealities), and channel coding/decoding. For large BSs the digital baseband also includes the power consumed by the serial link to the backbone network. Finally, platform control and MAC operation add a further power consumer (control processor).

The silicon technology significantly affects the power consumption $P_{\mathrm{BB}}$ of the $\mathrm{BB}$ interface. This technology scaling is incorporated into the power model by extrapolating on the International Technology Roadmap for Semiconductors (ITRS). The ITRS anticipates that silicon technology is replaced by a new generation every 2 years, each time doubling the active power efficiency but multiplying by 3 the leakage [13]. The increasing leakage puts a limit on the power reduction that can be achieved through technology scaling. Apart from the technology, the main parameters that affect the BB power consumption are related to the signal bandwidth, number of antennas and the applied signal processing algorithms. While the consumed power scales linearly with the bandwidth; MIMO signal detection scales more than linearly with the number of antennas.

Power Supply and Cooling: Losses incurred by DC-DC power supply, mains supply and active cooling scale linearly with the power consumption of the other components, and may be approximated by the loss factors $\sigma_{\mathrm{DC}}, \sigma_{\mathrm{MS}}$, and $\sigma_{\mathrm{cool}}$, respectively. Note that active cooling is only applicable to macro BSs, and is omitted in smaller BS types.

The breakdown of the BS power consumption at maximum load, $P_{\text {out }}=P_{\max }$, may be expressed as

$$
P_{\mathrm{in}}=N_{\mathrm{TRX}} \cdot \frac{P_{\mathrm{PA}}+P_{\mathrm{RF}}+P_{\mathrm{BB}}}{\left(1-\sigma_{\mathrm{DC}}\right)\left(1-\sigma_{\mathrm{MS}}\right)\left(1-\sigma_{\mathrm{cool}}\right)}
$$

where $N_{\text {TRX }}$ denotes the number of TRX chains of the considered BS type. Table I summarizes the state of the art power consumption of various LTE BS types as of 2010.

\section{B. BS Power Consumption at Variable Load}

In a conventional BS, the power consumption depends on the traffic load; it is mainly the PA power consumption that scales down due to reduced traffic load. This mainly happens
TABLE I

LTE Base STATION POWER CONSUMPTION AT MAXimum LOAD FOR DIFFERENT BS TYPES AS OF 2010.

\begin{tabular}{|c|c|c|c|c|c|c|}
\hline & Macro & Micro & Pico & Femto \\
\hline \multirow[t]{5}{*}{ PA } & $P_{\max }$ & {$[\mathrm{dBm}]$} & 46.0 & 38.0 & 21.0 & 17.0 \\
\hline & & {$[\mathrm{W}]$} & 40.0 & 6.3 & 0.13 & 0.05 \\
\hline & Back-off & {$[\mathrm{dB}]$} & 8.0 & 8.0 & 12.0 & 12.0 \\
\hline & PA Efficiency & [\%] & 31.1 & 22.8 & 6.7 & 4.4 \\
\hline & Total PA, $P_{\mathrm{PA}}$ & {$[\mathrm{W}]$} & 128.2 & 27.7 & 1.9 & 1.1 \\
\hline \multirow[t]{3}{*}{$\mathbf{R F}$} & $P_{\mathrm{TX}}$ & [W] & 6.8 & 3.4 & 0.4 & 0.2 \\
\hline & $P_{\mathrm{RX}}$ & {$[\mathrm{W}]$} & 6.1 & 3.1 & 0.4 & 0.3 \\
\hline & Total RF, $P_{\mathrm{RF}}$ & {$[\mathrm{W}]$} & 13.0 & 6.5 & 1.0 & 0.6 \\
\hline \multirow[t]{4}{*}{$\overline{\mathbf{B B}}$} & $\begin{array}{l}\text { Radio } \\
\quad \text { (inner Rx/Tx) }\end{array}$ & {$[\mathrm{W}]$} & 10.8 & 9.1 & 1.2 & 1.0 \\
\hline & $\begin{array}{l}\text { Turbo code } \\
\quad(\text { outer } \mathrm{Rx} / \mathrm{Tx})\end{array}$ & {$[\mathrm{W}]$} & 8.8 & 8.1 & 1.4 & 1.2 \\
\hline & Processors & [W] & 10.0 & 10.0 & 0.4 & 0.3 \\
\hline & Total BB, $P_{\mathrm{BB}}$ & [W] & 29.5 & 27.3 & 3.0 & 2.5 \\
\hline \multicolumn{2}{|c|}{$\overline{\text { DC-DC }, \sigma_{\mathrm{DC}}}$} & [\%] & 7.5 & 7.5 & 9.0 & 9.0 \\
\hline \multicolumn{2}{|c|}{ Cooling, $\sigma_{\mathrm{cool}}$} & [\%] & 10.0 & 0.0 & 0.0 & 0.0 \\
\hline \multicolumn{2}{|c|}{ Mains Supply, $\sigma_{\mathrm{MS}}$} & {$[\%]$} & 9.0 & 9.0 & 11.0 & 11.0 \\
\hline \multicolumn{2}{|c|}{ Total per TRX chain } & {$[\mathrm{W}]$} & 225.0 & 72.3 & 7.3 & 5.2 \\
\hline \multicolumn{2}{|c|}{ \# Sectors } & \# & 3 & 1 & 1 & 1 \\
\hline \multicolumn{2}{|c|}{ \# Antennas } & \# & 2 & 2 & 2 & 2 \\
\hline \multicolumn{2}{|c|}{ \# Carriers } & \# & 1 & 1 & 1 & 1 \\
\hline \multicolumn{3}{|c|}{ Total $N_{\text {TRX }}$ chains, $P_{\text {in }}[\mathrm{W}]$} & 1350.0 & 144.6 & 14.7 & 10.4 \\
\hline
\end{tabular}

when, e.g., the number of occupied subcarriers is reduced in idle mode operation, and/or there are subframes not carrying data. Naturally this scaling over signal load largely depends on the BS type; for macro BSs the PA accounts for 55-60\% of the overall power consumption at full load, whereas for low power nodes the PA power consumption amounts to less than $30 \%$ of the total.

Fig. 2 shows BS power consumption curves for a LTE system with $10 \mathrm{MHz}$ bandwidth and $2 \times 2 \mathrm{MIMO}$ configuration. Three sectors are considered for macro BSs, whereas omnidirectional antennas are used for the smaller BS types. While the power consumption $P_{\text {in }}$ is load dependent for macro BSs, and to a lesser extent for micro BSs, there is a negligible load dependency for pico and femto BSs. The reason is that for low power BSs, the impact of the PA is diminishing. Other components hardly scale with the load in a state of the art implementation; although some more innovative designs could lead to an improved power scaling at low loads. As can be seen in Fig. 2, the relations between relative RF output power $P_{\text {out }}$ and BS power consumption $P_{\text {in }}$ are nearly linear. Hence, a linear approximation of the power model is justified:

$$
P_{\text {in }}=N_{\text {TRX }} \cdot\left(P_{0}+\Delta_{\mathrm{p}} P_{\text {out }}\right), \quad 0 \leq P_{\text {out }} \leq P_{\max }
$$

where $P_{\max }$ denotes the maximum RF output power at maximum load, $P_{0}$ is the power consumption calculated at the minimum possible output power, assumed to be $1 \%$ of $P_{\max }$, and $\Delta_{\mathrm{p}}$ is the slope of the load dependent power consumption. Table II lists the parameters for the different BS types.

TABLE II

POWER MODEL PARAMETERS FOR DIFFERENT BS TYPES

\begin{tabular}{l|ccrl} 
BS type & $N_{\mathrm{TRX}}$ & $P_{\max }[\mathrm{W}]$ & $P_{0}[\mathrm{~W}]$ & $\Delta_{\mathrm{p}}$ \\
\hline Macro & 6 & 40.0 & 118.7 & 2.66 \\
Micro & 2 & 6.3 & 53.0 & 3.1 \\
Pico & 2 & 0.13 & 6.8 & 4.0 \\
Femto & 2 & 0.05 & 4.8 & 7.5
\end{tabular}



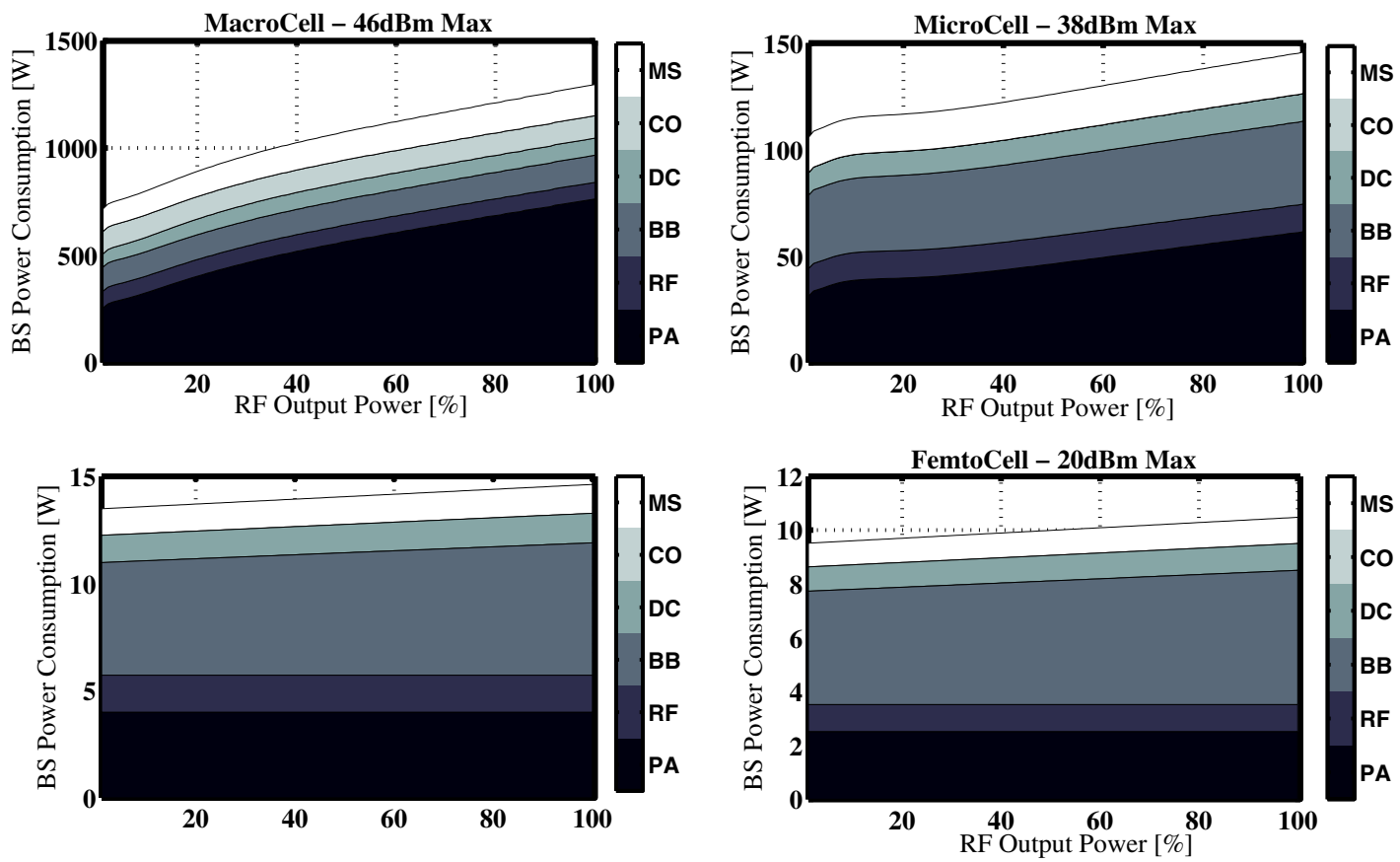

Fig. 2. Power consumption for various BS types as a function of the RF output power. An LTE system with $10 \mathrm{MHz}$ system bandwidth and $2 \times 2 \mathrm{MIMO}$ configuration is considered. Macro BSs employ 3 sectors per site. Legend: PA: power amplifier, RF: small signal RF transceiver, BB: baseband processor, DC: DC-DC converters, CO: active cooling (only applicable to macro BS), MS: mains power supply.

\section{TRAFFIC MODEL}

In order to provide a realistic analysis of the energy efficiency of wireless networks, it is essential to know the traffic demand to be served by the network. Thus, it is important to identify the spatial and temporal variation of the traffic demand both on large- and small-scale.

\section{A. Deployment Areas of Europe}

The average population density for different deployment areas in Europe is shown in Table III. Ratio of different deployment areas hardly depends on the particular countries of Europe; however, the Nordic countries (Finland, Norway and Sweden) and Russia make a big difference compared to the averages. We therefore choose to give the European average excluding the Nordic countries and Russia. Note that in central districts of a metropolis, the population density can exceed even 20,000 citizen $/ \mathrm{km}^{2}$, but due to their negligible covered area these are omitted from the presented model.

According to the current situation in Europe (see, e.g., [14]), the coverage of the latest mobile technologies is focusing on the population and not on the amount of area covered. That

TABLE III

Deployment AREas in Europe (ExCluding Nordic AND Russia)

\begin{tabular}{l|lll} 
Deployment & $\begin{array}{l}\text { Population } \\
\text { density } \\
\text { [citizen/km } / \mathrm{km}^{2} \text { ] }\end{array}$ & $\begin{array}{l}\text { Covered } \\
\text { area }[\%]\end{array}$ & $\begin{array}{l}\text { Normalized } \\
\text { mean peak } \\
\text { traffic load }\end{array}$ \\
\hline Dense urban & 3000 & $1 \%$ & $100 \%$ \\
Urban & 1000 & $2 \%$ & $33.3 \%$ \\
Suburban & 500 & $4 \%$ & $16.7 \%$ \\
$\begin{array}{l}\text { Rural } \\
\begin{array}{l}\text { Sparsely populated } \\
\text { \& wilderness }\end{array}\end{array}$ & 100 & $36 \%$ & $3.3 \%$ \\
& 25 & $57 \%$ & -
\end{tabular}

is, $2 \mathrm{G}$ area coverage is almost $100 \%$, while $3 \mathrm{G}$ coverage is below $40 \%$. This implies that the sparsely populated areas and the wilderness are only served by $2 \mathrm{G}$ networks. For instance, German regulation forces to serve "only" $90 \%$ of the population with wideband access [15], which practically allows to skip scarcely populated areas also for LTE deployment.

\section{B. Long-Term Large-scale Traffic Models}

The objective for the long-term large-scale traffic models is to determine the average served traffic on a certain time of day in a given deployment scenario. Abstracting the models from the current cell planning maps of Europe, the following methodology allows to deduce the daily traffic variations as the actual traffic demand of a given area:

1) define the average served data rates per subscriber;

2) determine the percentage of active subscribers;

3) given the population densities for the respective deployments, the scenario specific peak data rates per area unit in $\left[\mathrm{Mbps} / \mathrm{km}^{2}\right]$ can be derived;

4) finally, with the aid of a daily traffic profile the deployment specific data rates per area unit for a certain time of day is obtained.

1) Data rates per subscriber: The user generated data volume is tightly connected to operator policies and data subscriptions plans. Since the amount of traffic varies from country to country, we propose to define three traffic profiles, where the average traffic demand per subscriber is set to:

- high traffic profile sufficient to provide, e.g., HDTV for all active users, corresponding to $2 \mathrm{Mbps} / \mathrm{user}$;

- medium traffic profile sufficient to provide, e.g., SDTV for all active users, corresponding to $0.5 \mathrm{Mbps} / \mathrm{user}$; 
TABLE IV

Data TrafFic Peaks in the Respective Deployment AREAS

\begin{tabular}{r|rrr} 
Deployment & \multicolumn{3}{|c}{ Traffic } \\
& high & profile $\left[\mathbf{M b p s} / \mathbf{k m}^{2}\right]$ \\
mid & low \\
\hline Dense urban & 120.0 & 30.0 & 6.0 \\
Urban & 40.0 & 10.0 & 2.0 \\
Suburban & 20.0 & 5.0 & 1.0 \\
Rural & 4.0 & 1.0 & 0.2
\end{tabular}

- low traffic profile sufficient to provide, e.g., HQ Internet Radio with $\sim 100 \mathrm{kbps} / \mathrm{user}$, which represents intense data usage in current $3 \mathrm{G}$ networks.

We note that the above figures represent average traffic demands; typically strong temporal and geographical deviations with respect to these average values are experienced, e.g., one or two so-called heavy users may fully utilize a cell even for extended time periods.

2) Active subscribers: In today's networks $10-30 \%$ of the data subscribers are active in the busy/peak hours. According to the expectations towards wireless Internet services, the ratio of broadband data subscribers of the whole population will increase from year to year and in the most mature European markets may reach $25 \%$ by 2014; however, conservative expectations calculate with $10 \%$ as European average. ${ }^{2}$ The recommended aggregate reference values are as follows:

- the ratio of broadband data subscribers is $10 \%$ of the whole population;

- the number of active users in the busy/peak hours is $20 \%$ of the subscribers.

As a consequence, the number of active subscribers amounts to $2 \%$ of the whole population in the busy/peak hours, which roughly corresponds, e.g., to 10 users with active data connection in a typical urban cell.

3) Peak traffic demand per area: As the data volume per subscriber does not depend on the deployment scenario, the normalized traffic values in Table III are proportional to the population density. Given the population densities (see Table III), the traffic demand per subscriber (high, mid or low), and the number of active subscribers, the peak traffic demand per $\mathrm{km}^{2}$ of the considered deployment scenarios are listed in Table IV.

4) Served data traffic over time: Fig. 3 illustrates the typical daily variation in Europe (based on internal sources on traffic data and the Sandvine report [16]). The daily traffic variation are plotted relative to the peak traffic demand of a given deployment in Table IV. The same normalized traffic profile is assumed for all deployments.

The EARTH project found that the daily variation of the number of active users scales with the daily variation of the traffic. We therefore assume that the number of active users is scaled to match the traffic variations in Fig. 3, while the average rates per active user remain fixed.

\footnotetext{
${ }^{2}$ The amount of broadband subscribers correspond to subscriptions of at least $1 \mathrm{~GB}$ monthly cap, which are typically personal computer (PC) related today. However, smartphones and other mobile equipment could generate additional 10-25\% traffic in Europe; and even more in North America, due to much lower mobile PC traffic compared to Europe.
}

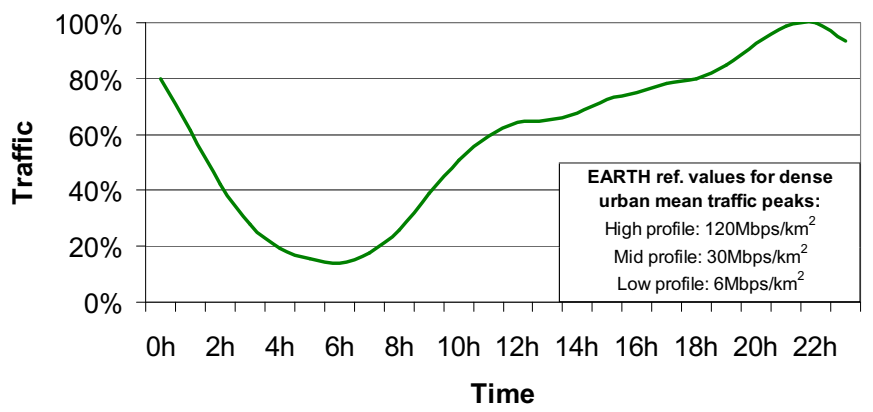

Fig. 3. Typical average daily data traffic profile in Europe.

Aggregating the traffic demands per active user over a whole month amounts to a traffic volume per subscriber of about 68 , 17 and $3 \mathrm{~GB} / \mathrm{month} /$ subscriber for the high, mid and low traffic profile, respectively. Note that these traffic demands are higher than that of current $3 \mathrm{G}$ networks, but should be considered as expectations for well-established mobile broadband markets beyond 2014. For instance, in today's mature markets a data volume of $1-3 \mathrm{~GB} / \mathrm{month} / \mathrm{subscriber}$ accounts as very intensive data usage [17-19].

\section{Statistical Short-Term Traffic Models}

In order to model the fluctuation of the traffic in short-time scale, the packet distribution generated by the different type of applications is modeled statistically. Since the same shortterm traffic models per active user should be applied in all deployment areas, the traffic demands in different deployments are derived from the differences in the corresponding user density figures. A detailed description of the traffic models can be found in [6].

\section{CAse Study: EnERgy EfFiciency of LTE}

For short-term, small-scale evaluations a macro-cellular network with regular hexagonal cell layout is implemented. 19 sites, each with 3 sectors, $10 \mathrm{MHz}$ bandwidth operating at $2.1 \mathrm{GHz}$ carrier frequency is assumed. Moreover, $2 \times 2$ MIMO transmission with adaptive rank adaption is assumed. The inter-site distance (ISD) for the dense urban and urban environments is set to $500 \mathrm{~m}$, whereas the ISD for suburban and rural areas is set to $1732 \mathrm{~m}$. The users are uniformly distributed, with population densities corresponding to the respective deployment scenarios. The simulation parameters are taken from 3GPP specifications [6].

The power per area unit $P / A$, expressed in $\left[\mathrm{kW} / \mathrm{km}^{2}\right]$, is depicted in Fig. 4. The power consumption increases with the served traffic in the network. In an urban scenario (see Fig. 4 (top)), with an ISD of $500 \mathrm{~m}$ corresponding to a coverage area of $0.2165 \mathrm{~km}^{2}$ per site, the power per area unit is around $4.15 \mathrm{~kW} / \mathrm{km}^{2}$ at low loads, whereas it approaches $5.1 \mathrm{~kW} / \mathrm{km}^{2}$ at high loads. For comparison, an empty network when only control channels are transmitted, but no user data, the power consumption equals $885 \mathrm{~W}$ per site, which corresponds to a power per area unit of $4.1 \mathrm{~kW} / \mathrm{km}^{2}$. In the (hypothetical) extreme case, when nothing at all is transmitted (i.e., no data and no control channels) so that the RF output power 


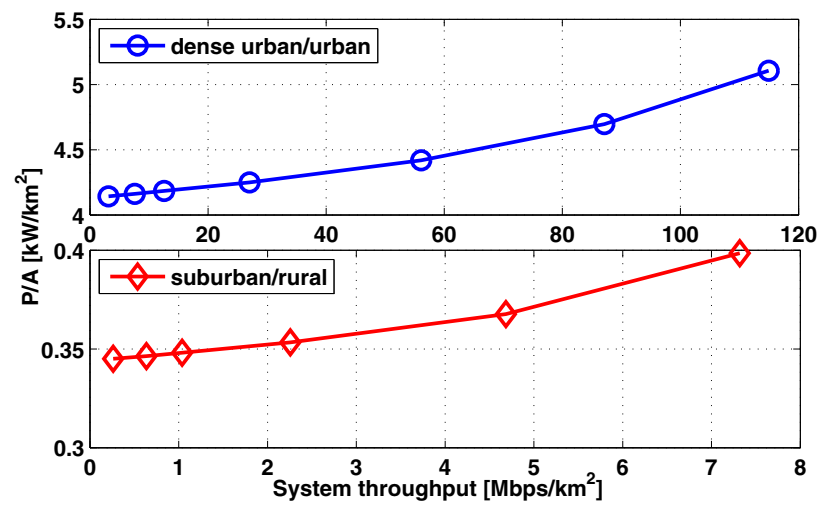

Fig. 4. Power per area unit, $P / A$, versus system throughput in dense urban/urban (top) and suburban/rural (bottom) scenarios. Notice the different scales in both $\mathrm{x}$ and $\mathrm{y}$-axis.

is $0 \mathrm{~W}$, we obtain $P / A=3.3 \mathrm{~kW} / \mathrm{km}^{2}$. The power consumption per area unit for suburban and rural areas, shown in Fig. 4 (bottom), is substantially lower, which is due to the increased ISD of $1732 \mathrm{~m}$, which corresponds to a coverage area of $2.6 \mathrm{~km}^{2}$. However, the system throughput per area unit decreases accordingly, due to the increased site coverage area.

In order to assess the expected performance of a countrywide area over a day, short-term, small-scale evaluations are combined with the long-term traffic models and the geographical distribution presented in Section 4. It is assumed that no coverage is provided in the sparsely populated and wilderness areas and hence, these areas are not included in the analysis. Moreover, the global traffic model in Section 4 is for the entire user population, whereas the short-term, small-scale evaluations are performed for a single carrier, which is served by a single operator. It is here assumed that the market share of the studied operator is $30 \%$ and that this operator also carries $30 \%$ of the total data traffic.

The outcome of the aggregation indicates that with the models and assumptions used in this evaluation, the average power per area unit is about $0.6 \mathrm{~kW} / \mathrm{km}^{2}$. This value is almost independent of whether high, medium, or low traffic density is assumed, i.e., the power consumption is mostly insensitive to the traffic load. One reason for this is that given the traffic and deployment models used here, the network typically operates in the very low load regime, where transmissions of control signals dominate over data. These evaluations highlight the fact that on average cellular networks are primarily providing coverage, and therefore mainly operate at low traffic loads.

\section{CONCLUSIONS}

In order to identify the key levers for energy savings the power consumption of mobile communication systems needs to be quantified. This includes sophisticated power models that map the radiated RF power to the supply power of a BS site, as well as traffic and deployment models that extend short-term small-scale evaluations to the country wide power consumption of a network over a whole day or week.
Numerical results reveal that for current network design and operation, the power consumption is mostly independent of the traffic load. This highlights the vast potential for energy savings by improving the energy efficiency of BSs at low load.

\section{ACKNOWLEDGMENTS}

This work has received funding from the European Community's $7^{\text {th }}$ Framework Programme FP7/2007-2013 under grant agreement $n^{\circ} 247733$ - project EARTH.

The authors gratefully acknowledge the invaluable insights and visions received from partners of the EARTH consortium.

\section{REFERENCES}

[1] International Telecommunication Union, "Worldwide mobile cellular subscribers to reach 4 billion mark late 2008," Press release, 2008.

[2] J. Malmodin, "The importance of energy in LCA for the ICT sector", in Proc. of SETAC Europe 14th LCA Case Study Symposium, Göteborg, Sweden, Dec. 2007.

[3] M. Gruber, O. Blume, D. Ferling, D. Zeller, M. A. Imran, and E. Calvanese-Strinati, "EARTH - Energy Aware Radio and Network Technologies," in Proc. IEEE Int. Symp. Personal, Indoor and Mobile Radio Communications (PIMRC), Cannes, France, 2008.

[4] J. T. Louhi and H.-O. Scheck, "Energy efficiency of cellular networks," in Proc. Int. Symp. Wireless Personal Multimedia Communications (WPMC), Lapland, Finland, 2008.

[5] L.M. Correia, D. Zeller, O. Blume, D. Ferling, Y. Jading, I. Godor, G. Auer, L. Van der Perre, "Challenges and Enabling Technologies for Energy Aware Mobile Radio Networks," IEEE Communications Magazine special issue on green radio, pp. 66-72, Nov. 2010.

[6] 3GPP TR 36.814 v9.0.0, "Further advancements for E-UTRA. Physical layer aspects (Release 9)," 3GPP, Technical Specification Group Radio Access Network, Mar. 2010.

[7] WINNER II, "D6.13.7: Test Scenarios and Calibration Cases Issues 2", Deliverable, IST-4-027756, December 2006.

[8] International Telecommunication Union, Report ITU-R M.2134, "Requirements related to technical performance for IMT-Advanced radio interface(s)," 2008. http://www.itu.int/dms_pub/itu-r/opb/rep/R-REP-M. 2134-2008-PDF-E.pdf

[9] S.C. Cripps, "RF Power Amplifiers for Wireless Communications", Artech House Microwave Libreary, 2nd Edition, 2006.

[10] J. Xu, "Practical Digital Pre-Distortion Techniques for PA Linearization in 3GPP LTE", Agilent Technologies, 2010.

[11] W. Kim, S.P. Stapleton, J.H. Kim and C. Edelman. "Digital PreDistortion Linearizes Wireless Power Amplifiers", IEEE Microwave Magazine, Sep. 2005.

[12] B. Debaillie, A. Giry, M.J. Gonzalez, L. Dussopt, M. Li, D. Ferling, V. Giannini, "Opportunities for Energy Savings in Pico/Femto-cell Base-Stations," in Proc. Future Networks and Mobile Summit, Warsaw, Poland, 2011.

[13] S. Borkar, "Design Challenges of Technology Scaling," IEEE Micro, vol. 19, no. 4, pp. 23-29, July 1999.

[14] National Media and Infocommunications Authority Hungary, "Report on mobile internet coverage," 2010. http://www.nmhh.hu/dokumentum. php?cid=25074

[15] "Entscheidung der Präsidentekammer der Bundesnetzagentur fur Elektrizität, Gas, Telekommunikation, Post und Eisenbahnen" (in German), October 2009. http://www.bundesnetzagentur.de/cae/servlet/contentblob/ 138464/publicationFile/2807/PraesKammerEntschg_Id17404pdf.pdf

[16] Sandvine, "Mobile Internet Phenomena Report", 2010. http://www. sandvine.com/downloads/documents/2010 Global Internet Phenomena Report.pdf

[17] Akamai, "State of the Internet" report, 2010. http://wwwfp.akamai.com/ dl/whitepapers/akamai_state_of_the_internet_q1_2010.pdf

[18] National Media and Infocommunications Authority of Hungary, "Report on mobile data usage", 2010. http://www.nhh.hu/dokumentum.php?cid= 24769

[19] The Swedish Post and Telecom Agency, "Telecommunication Markets in the Nordic Countries", 2010. http://statistik.pts.se/pts1h2010e/download/ PTS_ER_2010_26_Svensk_Telemarknad_2010_1h_en.pdf 